\title{
Letters from the Lactide Frontiers 2019
}

H. Haller, F. Sander, B. Hartmann

BG Trauma Hospital Berlin, Burn Center with Plastic Surgery, HLMedConsult

Polylactic Membranes (PLMs) based on Polymers from mixtures of lactic acids have been used in burns treatments for nearly twenty years. They are established as a well-recognized treatment method that allows for short healing time, low complications, excellent cosmetic results in superficial, deep partial thickness and even small full thickness burns [1-9] under reduced pain. This poster will try to explain the basic and the "Lactate Effect" in burns treatment and correlate this with implications for the clinical use.

We know that polylactides degenerate to chemical fragments releasing lactate and lactic acid [10]. Lactate and lactic acid serve as an energy source for the cells $[10,11]$. Both substances can permeate the cell membranes either due to their molecule size or to active transport by the monocarboxylate transporter (MCT) protein shuttle system. Inside of the cell, lactate can serve as an energy source via the Cori cycle or lactate can oxidize to pyruvate (via lactate dehydrogenase), which can then oxidize to acetyl-CoA and fuels the TCA cycle in mitochondria producing the metabolic products carbon dioxide, water, and NADH which provides the energy-rich NAD.

Lactate and pyruvate act as potent antioxidants $[10,12-14]$ within the cells. Medical literature describes the role of oxidative stress in an increasing manner. Lactate acts on cytokines and increases TGF- $\beta$ activation, Hypoxia Inducible Factor, VEGF, and others as many studies showed [15-18].

Polylactic membranes act as activators in wound healing and support curing by supplementation of energy. Telomeres conservation will contribute to a reduction of cell-aging and eternization, maintaining the rate of duplications and the quality of the skin. TGF- $\beta$ affects keratinocytes, completing the activation status for healing. In dermal tissue, it increases fibroblast growth, collagen, and extracellular matrix synthesis as well as neovascularization. It acts as a radical scavenger as well. Clinical findings bolster these laboratory results.

Using these effects enables you to use polylactides as a toolbox for a broad range of indications.

\section{Surface and structure effects}

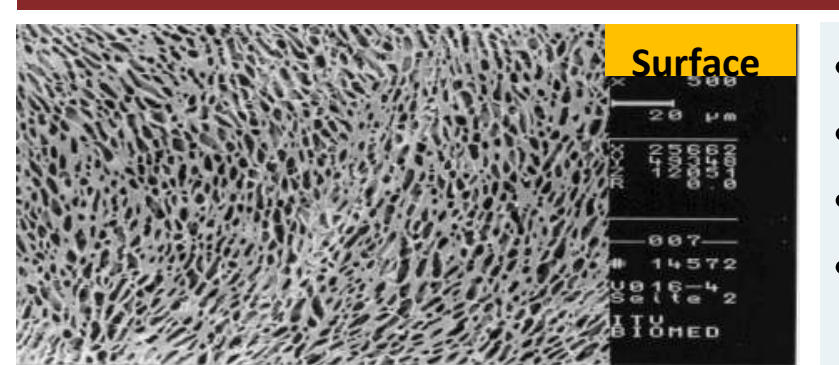

Wake, Patrick, and Mikos 1994: Positive correlation between pore size and formation of fibrous tissue was found.

- Yannas and Burke 1980 mention the significance of pore size for cell ingrowth.

- Pinney 2000 described the effect of fibroblasts seeded on a 3D scaffold on vasculogenesis.

- Ring et al. demonstrated in 2011 that structure as bimodal foam influences the amount of vasculogenesis positively: VEGF mRNA was expressed in a 22 fold amount in the three-dimensional structure greater than a monolayer

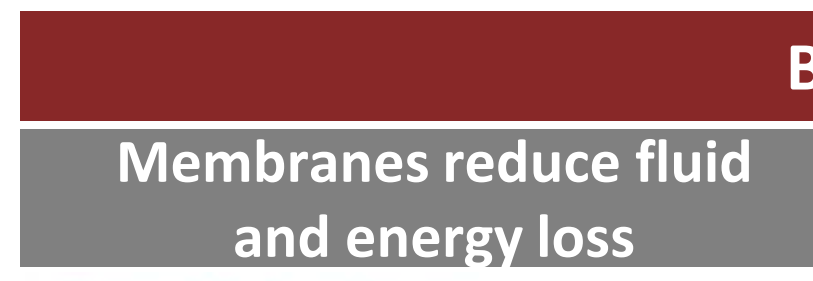

Permeability to water vapor

Permeability to water vapor

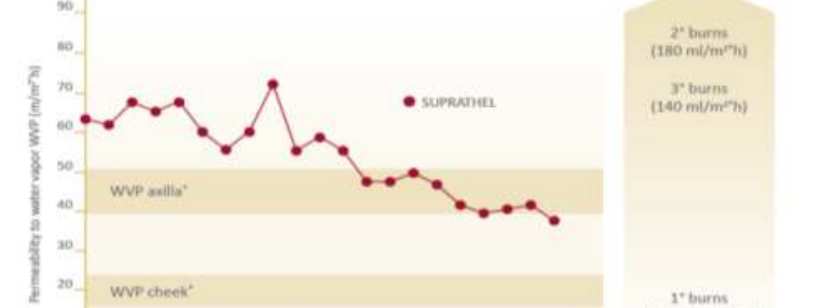

\section{Barrier effects}

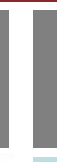

Membranes protect
against Superinfection

PLM protects against Infection even in DPT burns with non-wound associated infectionsNo difference in healing time

$\begin{array}{cccccc}\text { Grp } & \text { Mean } & \text { Std. } & \text { SEM } & \begin{array}{c}\text { Media } \\ n\end{array} & n \\ \text { Inf. } & 7,82 & 4,03 & 0,65 & 7 & 39 \\ \text { No Inf. } & 8,17 & 5,24 & 0,66 & 7 & 64\end{array}$

Hartmann, Sanders, Haller ABA 2019
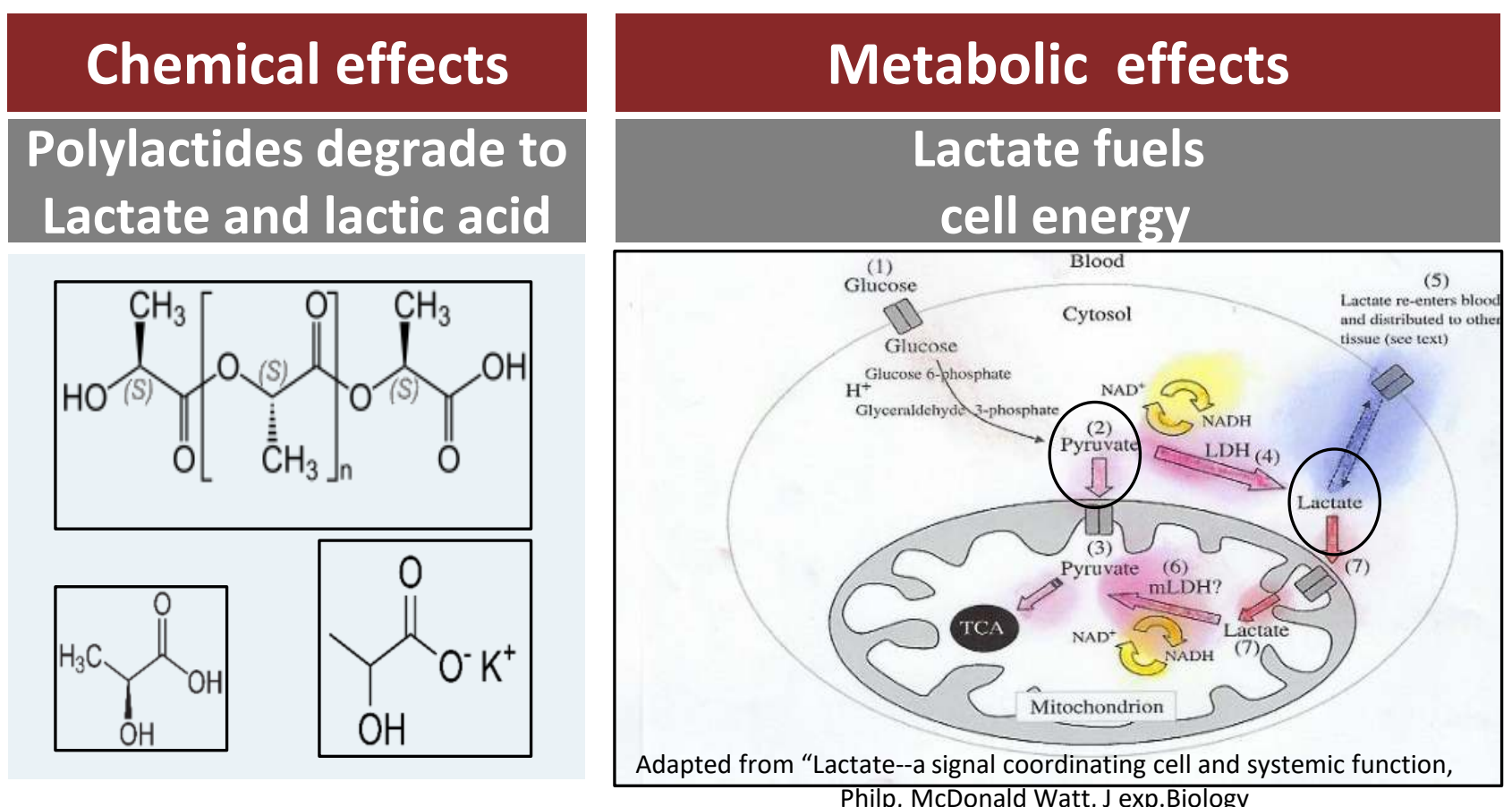

Wound healing effects

"Externally applied lactate simulates hypoxic conditions under normal oxygen pressure" Philp A, Macdonald AL, Watt PW. J Exp Biol 2005;208:4561-75

Lactate acts as a signal molecule promoting wound healing

\section{Lactate acts on dermal tissue}

\section{Lactate activates}

- TGF- $\beta$ and VEGF even without the HIF pathway.

An addition of extracellular lactate leads to

- significant short-term elevation of interleukin-1b, a long-term elevation of VEGF and transforming growth factor- $\beta 1$, and a $50 \%$ elevation in collagen deposition

\section{Fibroblast proliferation (Penn 2012)}

ECM formation like hyaluronan (Stern 2002)

Collagen synthesis and deposition ( Penn 2012)

Angiogenesis (Ring 2011, Porporato 2012)
Lactate acts on epidermal tissue via TGF

IL-1

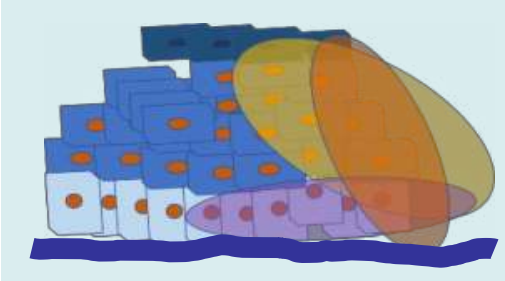

TNF - activation state

Dedifferentiation

ECM creation

Cell motion, forming of

filopodia

Proliferation

Redifferentiation

reverting keratinocytes to basal phenotype

\section{Systemic effects}

\section{Acts as Free Radical Scavenger}

Use in moist Desquamation due to Radiation: Rothenberger 2016

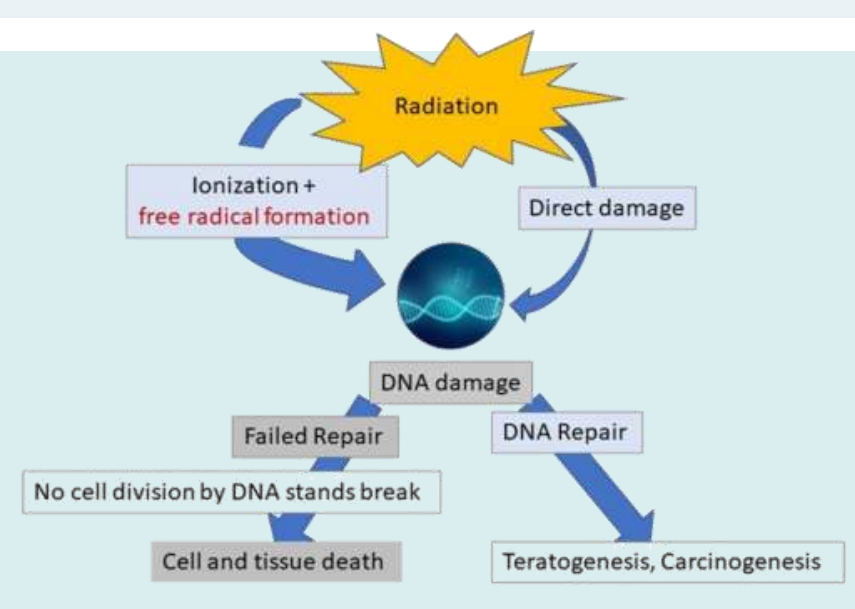

Reduces oxidative stress

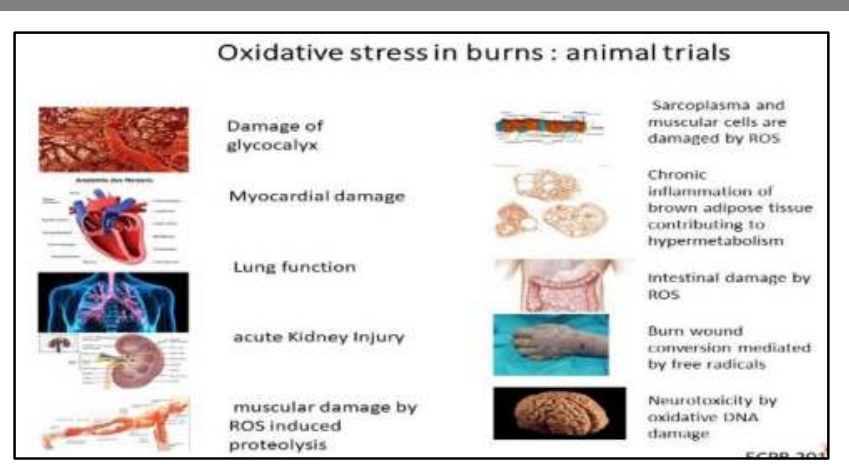

PLM versus HFAG: Total oxidant and Total Antioxidant Capacity
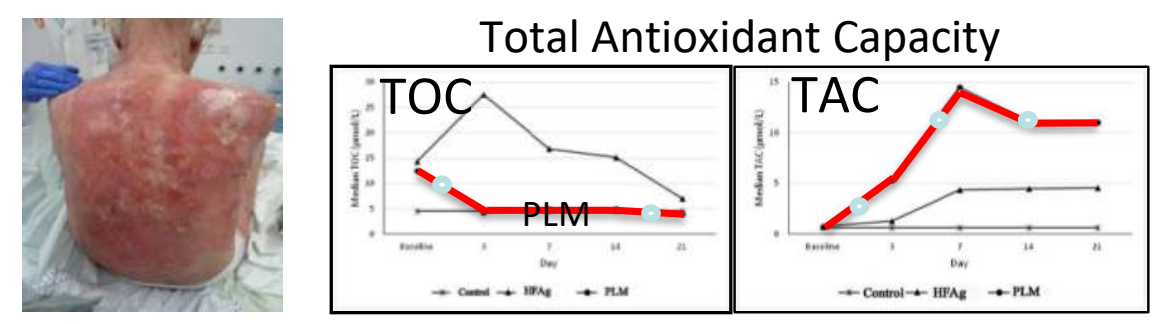

Reduces systemic inflammatory response

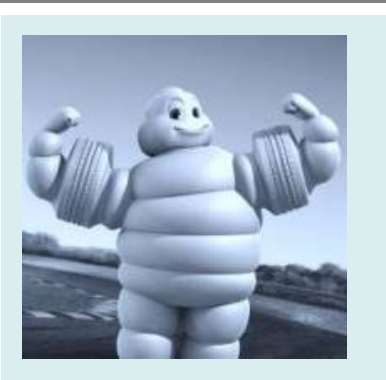

Fluid creep

Stabilization of glycocalyx Scar development
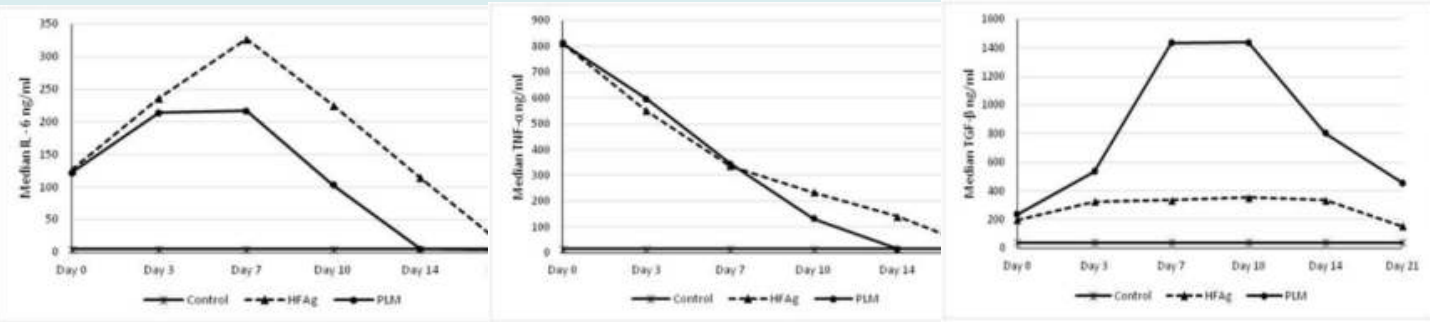

Gürünlüoğlu K¹, Demircan M¹ , Taşçı A ${ }^{1}$, et al 2019 